\title{
Interaural time vs. interaural intensity in a lateralization paradigm
}

\author{
WILLIAM A. YOST, DAVID C. TANIS, DONALD W. NIELSEN, and BYRON BERGERT \\ Communication Sciences Laboratory, University of Florida, Gainesville, Florida $\$ 2611$
}

\begin{abstract}
In a two-interval lateralization procedure, observers judged whether a stimulus presented with an interaural intensive difference was right or left in lateral space of the same stimulus presented with only an interaural temporal difference. The stimuli were pure tones of 500 and $1,000 \mathrm{~Hz}$ and $1,000-\mathrm{Hz}$ low-pass noise. All stimuli were presented at both 65 and $55 \mathrm{~dB}$ SPL. For each of several values of interaural time (ranging from 0 to 1,000 microsec across all stimuli), a function was determined which related proportion of "right" relative position judgments to the value of the interaural intensive difference. The intercepts of these functions indicated that a progressively smaller amount of interaural intensive difference was required for the two stimuli to occupy a similar lateral location as the interaural temporal difference was increased. The slopes of the function suggested that the images associated with larger values of the interaural temporal differences are less distinct and blend together more than the images associated with small values of the temporal difference. Thus, the procedure provided a means for comparing the lateral location of images produced by interaural differences of time and intensity.
\end{abstract}

Many investigators have studied how interaural temporal differences (ITD) and interaural intensive differences (IID) alone mediate one's ability to lateralize a sound source. Except for investigations of the "binaural frading ratio," however, little research has been devoted to studying the interaction of the ITD and the IID in lateralization. The binaural trading ratio (Green \& Henning, 1969) is the ratio of the ITD to the IID when an intensity-displaced image is brought back to midline with an interaural temporal difference. This procedure has been assumed capable of accurately determining the interaction of interaural time and intensity in lateralization.

The results of the binaural trading ratio experiments are, however, not easily interpreted. Hafter and Jeffress (1968) showed that, for pure tones, observers often hear two lateral images in the binaural trading ratio experiment: one image associated with the ITD, the other with the IID. Furthermore, they suggested that the wide range of trading ratios reported in the literature are a result of the inability to obtain a "centered image." Hafter and Carrier (1972) and Hershkowitz and Durlach (1969b) demonstrated that, although an observer might report an image at midline, no combination of the IID and the ITD could be obtained which yielded an image indiscriminable from that produced by a tonal

This research was supported in part by a National Institutes of Heaith (NIH) program project grant, an NIH training grant, an NIH research grant, a University of Florida research grant, and a grant from the Northeast Regional Data Center of the University of Florida. D. C. Tanis' present address is: Neurophysiology/Biophysics. VA Hospital, 150 Muir Road, Martinez, California 94553. D. W. Nielsen's present address is: Otological Research Laboratory, Department of Otolaryngology, Henry Ford Hospital, 2799 West Grand Boulevard, Detroit, Michigan 48202. stimulus with no interaural differences. Thus, the difficulty in interpreting these binaural trading ratio experiments seems to lie in their procedure which required one "centered image."

The purpose of the present experiment was to investigate the interaction of interaural time and intensity in a paradigm which avoids the "centered image" problem. Rather than cancelling the effects of the IID with the ITD, as was attempted in the binaural trading ratio experiments, we wanted to determine which value of the IID produced a lateral image with the equivalent location of that produced by an ITD. In order to determine these values, we used a left-right lateral judgment procedure similar to that used by Sayers and Cherry (1957) and Molino (1974).

\section{METHOD}

The left-right lateralization method task is basically a two-interval task. On each trial, two stimuli were presented: the first stimulus usually contained only an interaural temporal difference, and the second stimulus only an interaural intensive difference. The observer indicated whether the second stimulus (IID) appeared to the left or right in lateral space of the first stimulus (ITD). For each value of the ITD, a function was obtained which related the proportion of "right" relative position judgments to the IID.

The stimuli in this experiment were $200 \mathrm{msec}$ in duration and were shaped with a $10-\mathrm{msec}$ rise-decay time. Two tones, 500 and $1,000 \mathrm{~Hz}$, and a 1,000-Hz low-pass White Gaussian noise $(1,000-\mathrm{Hz}$ high-pass cutoff, with a $24-\mathrm{dB} /$ oct. roll-off provided by a Kron-Hite filter, Model 3100) were presented at two overall levels, 55 and $65 \mathrm{~dB}$ SPL (overall level refers to the true RMS level of the noise after filtering). The stimuli were presented through binaurally matched TDH-39 headphones to observers seated in a single-walled IAC booth. Four observers served as subjects. All observers were practiced listeners in lateralization tasks.

Figure 1 is a diagram of the stimulus sequence and the lateralized images perceived. The first of the two intervals 

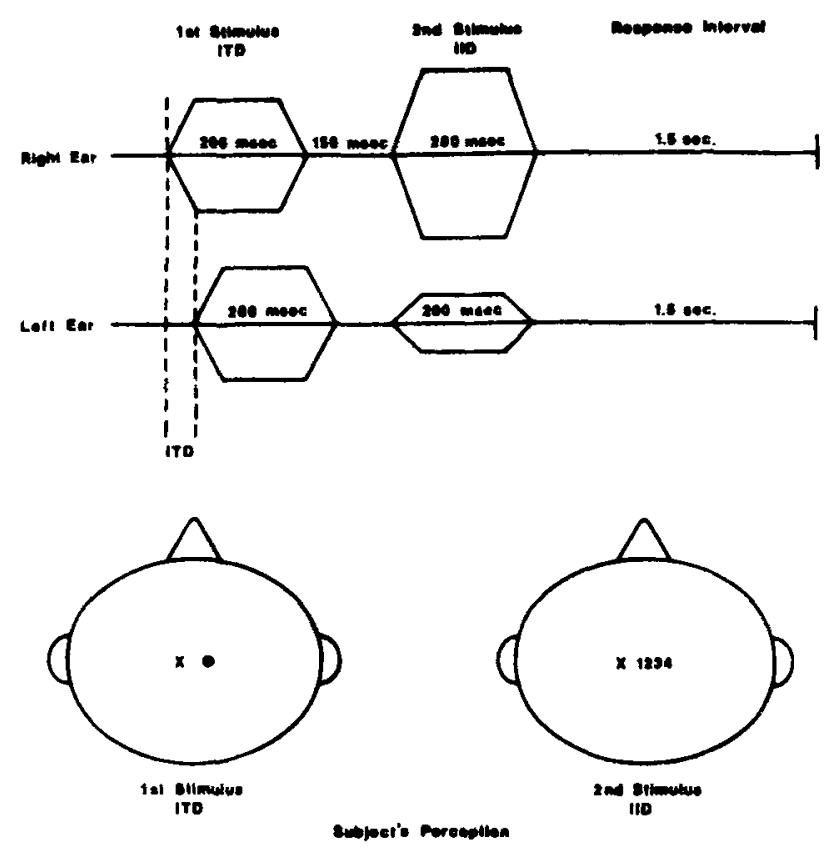

Figure 1. The stimuli's temporal sequence, ITD, and IID are shown schematically in the top of the figure. The first stimnlus pair has an ITD and creates an image lateralized to some extent as shown by the solid circle in the bottom of the figure. The second stimulus has only an IID and creates an image in one of the four positions illustrated by the numbers in the bottom of the figure. The sabject's task is to say whether the image of the second stimulus appeared to the right or left of the first.

contained a stimulus presented with only an interaural temporal difference (Ad-Yu delay line Model 801F). ${ }^{1}$ The ITD was always presented so that the right ear led in time. Following a 150 -msec pause, the same stimulus was presented with an interaural intensive difference in the second interval. The observer responded either: (1) "the second stimulus appeared to the right" or (2) "the second stimulus appeared to the left". of the first stimulus. The observer had $1.5 \mathrm{sec}$ in which to respond. One light was used to indicate the beginning of each trial, and another light to define the response interval. The second stimulus (IID) was presented randomly at one of four predetermined values, each separated by $2 \mathrm{~dB}$. These values were presented so that the right ear was incremented while the left ear was decremented by equal amounts. Thus, all stimuli could be presented to the two ears at the same average intensity. This allowed approximately the same loudness to be presented during both intervals.

A preliminary experiment was used to obtain an estimate of the ranges of the IID which would be required for each value of the ITD and for each observer. For each value of the ITD and for each observer, the IID was increased until for at least 40 trials the observers was designating some of the four second-interval stimuli (stimulus presented with the IID) as being "right" of the first interval stimuli. These values of the IID were then used throughout the rest of the experiment.

In the main experiment, at least 20 trials were presented at each of the four values of the IID. The collection of these data began after the observer was presented a few practice trials. Thus, the truly random nature of the four IID presentations and the presence of practice trials required between 95 and 140 actual trials for each value of the ITD.

Occasionally, test trials were presented to check the stability of this left-right judgment procedure. Values of the IID either 1-2 dB or $6 \mathrm{~dB}$ different from those actually used were introduced. For the conditions in which only a 1-2-dB change in the IID was introduced, the observers produced the same function relating proportion of "right" judgments to the IID as during the regular procedure. That is, for the test trials, a 2-dB change might yield a higher proportion of "right" judgments than during the regular procedure, but the function relating proportion of "right" judgments to IID remained approximately the same. When a change of $6 \mathrm{~dB}$ was introduced, the observers responded with either $100 \%$ "right" or $100 \%$ "left" judgments, depending on the direction of the 6-dB change. These results indicate that this procedure produced a relatively stable judgment criterion in the observers.

For each value of the ITD, a function was obtained which related the proportion of "right" relative position judgments to the IID. The slope of these functions and the $50 \%$ value of the proportion of "right" judgments were used to describe the interaction of the interaural temporal and intensive differences used in this experiment.

\section{RESULTS}

The data were averaged across the four observers by employing a curve-fitting procedure. First, the results from each observer and at each value of the ITD were plotted as the proportion of "right" judgments vs. the IID. These functions revealed relatively large intersubject variability. The variability was expressed in terms of the total amount of the IID each observer used for the $108^{\circ}$ change in the ITD. The range was from 7 to $11 \mathrm{~dB}$. That is, at $108^{\circ}$, some observers indicated that the two images were in the same spatial location when the IID was approximately $7 \mathrm{~dB}$, while other observers used approximately $11 \mathrm{~dB}$. Although there was a 4-dB intersubject variability, all observers showed the same systematic change in the form of the functions as the ITD was varied.

Since the functions appeared to have the sigmoidal shape of the cumulative normal distribution, the proportion of "right" judgments was transformed to $z$ scores by using a table of areas under the normal distribution curve (scores of $0 \%$ or $100 \%$ proportion of "right" judgments were not transformed to a $z$ score). The transformed functions were fitted by a straight line using a least square routine (in all cases, the correlation coefficient for the linear best fits was greater than 0.94 ). The best fitting values of the slope and intercept constants for each function were then averaged across observers to relate $z$ scores for "right" judgments to the IID.

Figure 2 shows the average functions relating $z$ scores to the IID for the six stimulus conditions. Positive $z$ scores represent proportion of "right" judgments of greater than $50 \%$, while negative values are for proportion of "right" judgments less than $50 \%$. The proportion of "right" judgments is shown on the right ordinate of each panel.

Investigation of Figure 2 reveals that there are only small differences among the four tonal conditions (Panels A-D). To confirm this observation, an analysis of variance of the slope and intercept values 


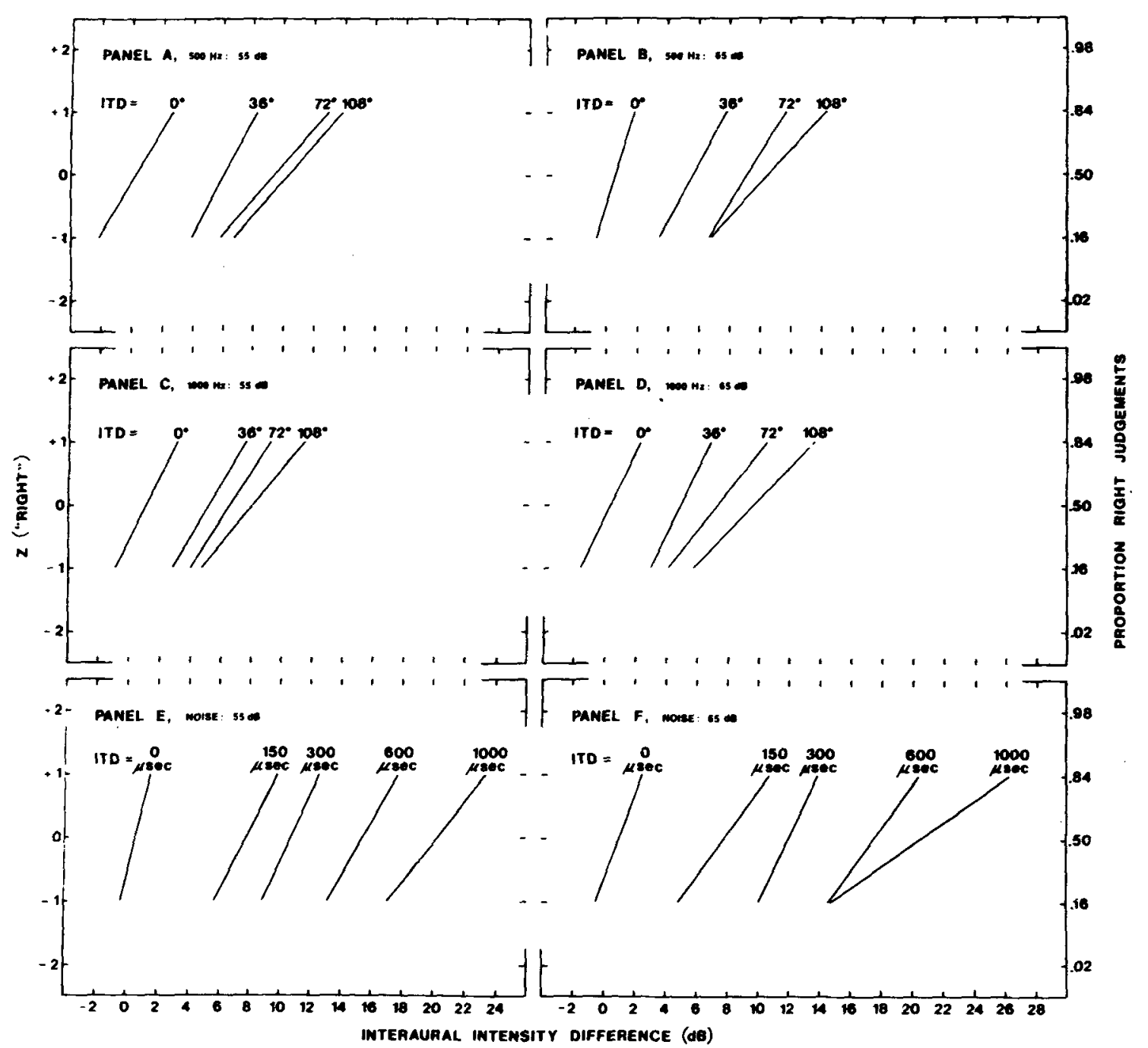

Figure 2. The $\mathrm{z}$ scores for the proportion of "right" jadgments [z("right")] as a function of the interaural intengive difierence in decibels. The parameter on each curve is the interaural temporal difference of the first stimulus in the bateral left-right judguent task. Panel $A$ represents the 500-Hz, 55-dB SPL condition; Panel B, the 500-Hz, 65-dB SPL condition; Panel C, the 1,000-Hz, 55-dB SPL condition; Panel D, the 1,000-Hz, 65-dB SPL condition; Panel E, the 1,000-Hz low-pass noise, 55-dB SPL condition; and Panel F, the 1,000-Hz low-pass noise, 65-dB SPL condition. The data were avernged across all four observers.

was used. The main purpose of the analysis of variance was to determine if we could average the data across frequency and intensity, in order to make the discussion of the results less complicated. Since little information is available concerning the sampling distributions for these functions, it is not clear as to what statistical analysis is most appropriate or if the slopes and intercepts should be treated independently. We believe, however, that if the slope and intercept values do not yield statistically significant differences for frequency and intensity, then it is probably appropriate to average across frequency and intensity. Thus, the main effects in each analysis were the four values of the ITD, the two frequencies, and the two levels of overall intensity. In the slope analysis, the ITD was significant at the .05 level $(\mathrm{F}=$ 15.86, $\mathrm{df}=3,48$ ); the ITD was also significant at the

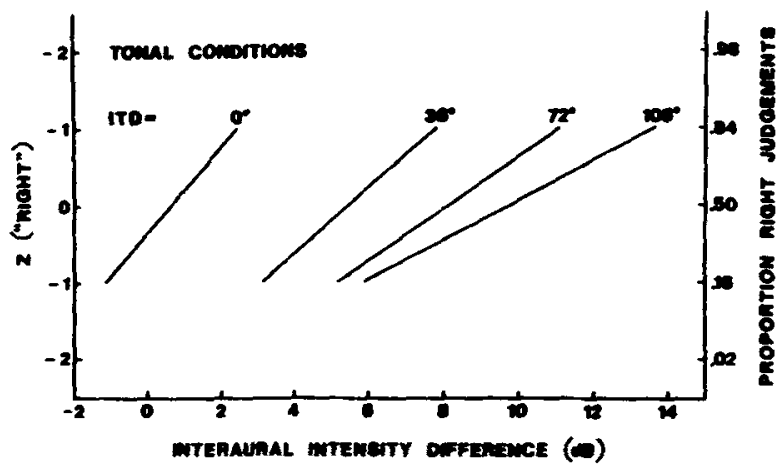

Figure 3. The $z$ scores for the proportion of "right" judgments [z("right")] as a function of the interaural intembe difference bn decibels. The parameter is the interaural temporal difference in degrees. The data are avernged across tonal frequency, tond intensity, and observers. 


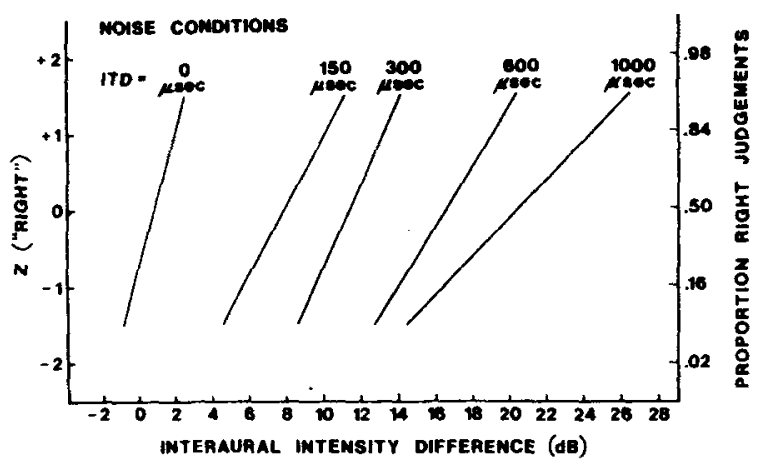

Figure 4. The $\mathrm{x}$ scores for the proportion of "right" judgments $[z$ ("right")] as a function of the interaural intensive difference in decibels. The parameter is the interaural temporal difference in microseconds. The data were averaged across the overall level of the 1,000-Hz low-pass noise and across observers.

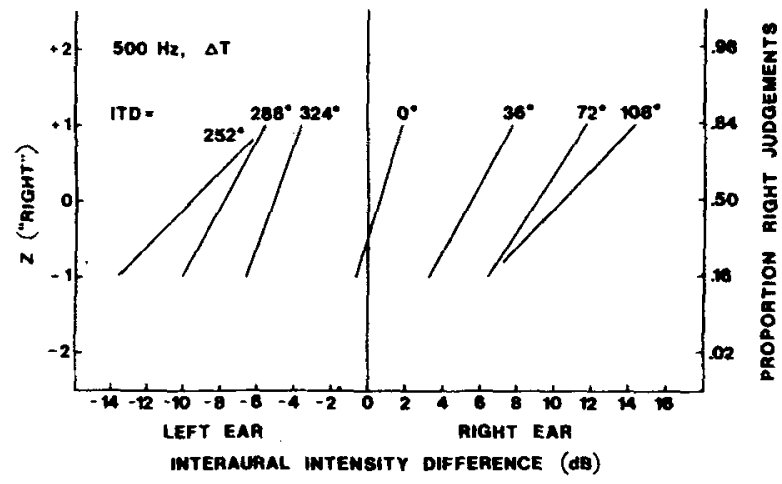

Figure 5. The z scores for the proportion of "right" judgments $[x$ "right")] as a function of the interaural intensive difference in decibels. The parameter is the interaural temporal difference in degrees. The data are for the $500 \cdot \mathrm{Hz}, 65 \cdot \mathrm{dB}$ SPL condition and are averaged acmss two of the four observers.

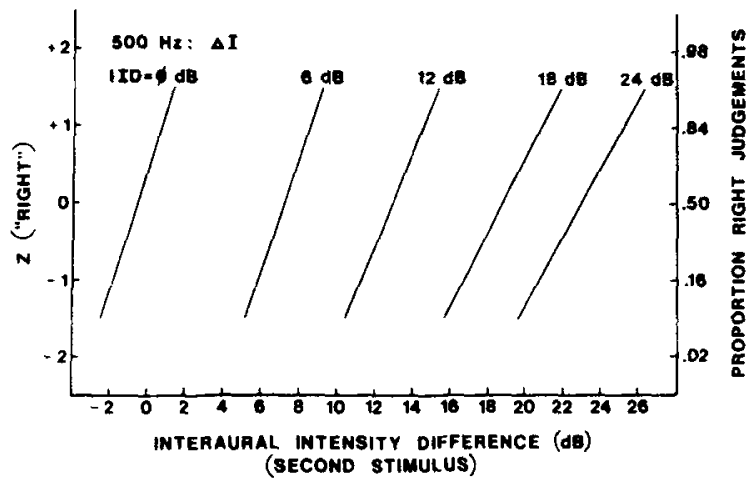

Figure 6. The $\mathrm{z}$ scores for the proportion of "right" judgments as a function of the interaural intensive difference. The parameter is the interaural intensive difference of the first stimulus in the lateral left-right judgment task. The data are for the 500-Hz, 65-dB SPL condition and are the average of two of the four observers.
.05 level $(\mathrm{F}=45.69 . \mathrm{df}=3.48)$ in the analysis of the intercepts. No other main effects or interactions proved to be statistically significant. Therefore, the data were averaged across intensity and frequency, as well as across observers. to more clearly demonstrate the form of the functions relating $\mathrm{z}$ scores to the IID for each value of the ITD. The averaged data are displayed in Figure 3.

Another analysis of variance was computed for the noise conditions. Again, in order to justify averaging of the data, we computed one analysis for the slope constants and other analysis for the intercept constants. The main effects of this analysis of variance were the ITD and overall level. The only significant $F$ ratios were obtained for the ITD. For the slope constant, the ITD was significant at the .05 level ( $F=$ 9.23, $\mathrm{df}=4.30$ ), and for the intercept constant, the ITD was significant at the .05 level $(F=88.33$, df $=$ 4.30). Thus, the noise data (shown in Figure 4) were averaged across overall level and observers.

All of the data in Figures 2 and 3 were obtained for ITDs of less than $180^{\circ}$. For the ITDs greater than $180^{\circ}$, symmetric functions should be obtained, but the images should be on the left side of the head. To test this assumption, two of the four observers listened to the $500-\mathrm{Hz}, 65-\mathrm{dB}$ SPL tone in the left-right lateralization task when the ITD was greater than $180^{\circ}$. Their averaged data, shown in Figure 5, clearly support the assumption of symmetry.

A final condition was tested using this lateralization procedure. In this condition, the two observers who were used to obtain the data for Figure 5 were asked to compare two stimuli presented with an IID. In order to make the two stimuli different, the first value of the IID was presented so that the level at the left ear remained constant while the level at the right was increased. The IID for the second stimulus in the task was generated as in the main experiment: the left-ear stimulus was decreased in level, while the stimulus at the right ear was increased by an identical value. Thus, as the IIDs for both stimuli were increased, the first stimulus appeared to increase in loudness, while the second stimulus remained approximately constant in loudness. The averaged results for the $500-\mathrm{Hz}$, 65-dB SPL condition are shown in Figure 6. As can be seen, a $6-\mathrm{dB}$ increase in the IID of the first stimulus required a $6-\mathrm{dB}$ increase in the IID of the second stimulus in order for the observers to make consistent left-right judgments. An analysis of variance of the data in Figure 6 revealed that the slope differences were not statistically significant at the .05 level.

\section{DISCUSSION}

Two main aspects of these data will be discussed: (1) the value of the IID which yields a $z$ score of 0 for a given ITD, and (2) the slope of the z-scored functions at each value of the ITD. 
The value of the IID which yields a $\mathrm{z}$ score of 0 is the one in which the observer would state that the second stimulus (IID) was judged only $50 \%$ of the time to be right (or left) of the first stimulus. This value of the IID is considered to equate the two stimuli in terms of location in lateral space. Figure 7 is a plot of the values of IID (second stimulus) which yielded a $\mathrm{z}$ score of 0 as a function of the ITDs in Panel A and Panel B and as a function of the IID in Panel C. Panel A represents the tonal data of Figure 3, Panel $B$ the noise data of Figure 4, and Panel $\mathrm{C}$ the $500-\mathrm{Hz}$ data of Figure 6 . The data in Figure 7 Panels $A$ and $B$, therefore, describe the binaural trading ratio in terms of the values of the interaural differences required for images to occupy equivalent lateral locations.

The data from Figure 2. Table 1, and the analysis of variance indicate that the lateral judgments for 500 and $1,000 \mathrm{~Hz}$ are similar if the ITD is expressed in terms of the interaural phase rather than in terms of the interaural temporal difference. This is consistent with results from interaural temporal discrimination experiments (see Yost, 1974, for review) and with results from experiments in which observers directly indicated where in lateral space images associated with ITD are perceived (see Sayers, 1964). Observers maintain approximately constant performance in lateral discrimination procedures as a function of changes in frequency when the ITD is expressed in values of interaural phase. Observers also indicate that lateral images associated with different frequencies are at the same lateral location when the ITD is presented in terms of an interaural phase difference.

Panels $A$ and $B$ in Figure 7 show that for the noise stimulus, $1,000 \mu \mathrm{sec}$ of the ITD requires a larger value of the IID for a $z$ score of 0 than does $108^{\circ}$ of the ITD for the tones. This is probably due to the fact that $1,000 \mu \mathrm{sec}$ is close to the limit for the ITD which yields a change in lateral position of a noise, whereas $180^{\circ}$ is probably the limiting value of the ITD for tones. Thus, an additional $72^{\circ}$ of the ITD could have been presented for the tones before the limiting value of the ITD was reached. Also, $108^{\circ}$ corresponds to only $600 \mu \mathrm{sec}$ of ITD for the $500-\mathrm{Hz}$ tone and $300 \mu \mathrm{sec}$ of ITD for the $1,000-\mathrm{Hz}$ tone.

The data in Panel $\mathrm{C}$ of Figure 7 show that changes in the IID of one stimulus require an equal interaural intensive change in the other stimuli for the two lateral images to occupy similar lateral locations. This result was obtained even though the two stimuli were presented at very different intensities. Thus, two stimuli presented with the same value of IID are perceived as being in the same spatial location even though they may not be of the same perceived loudness.

Not only do the IIDs required for a $\mathrm{z}$ score of 0 decrease as the ITD increases, but the slopes of the

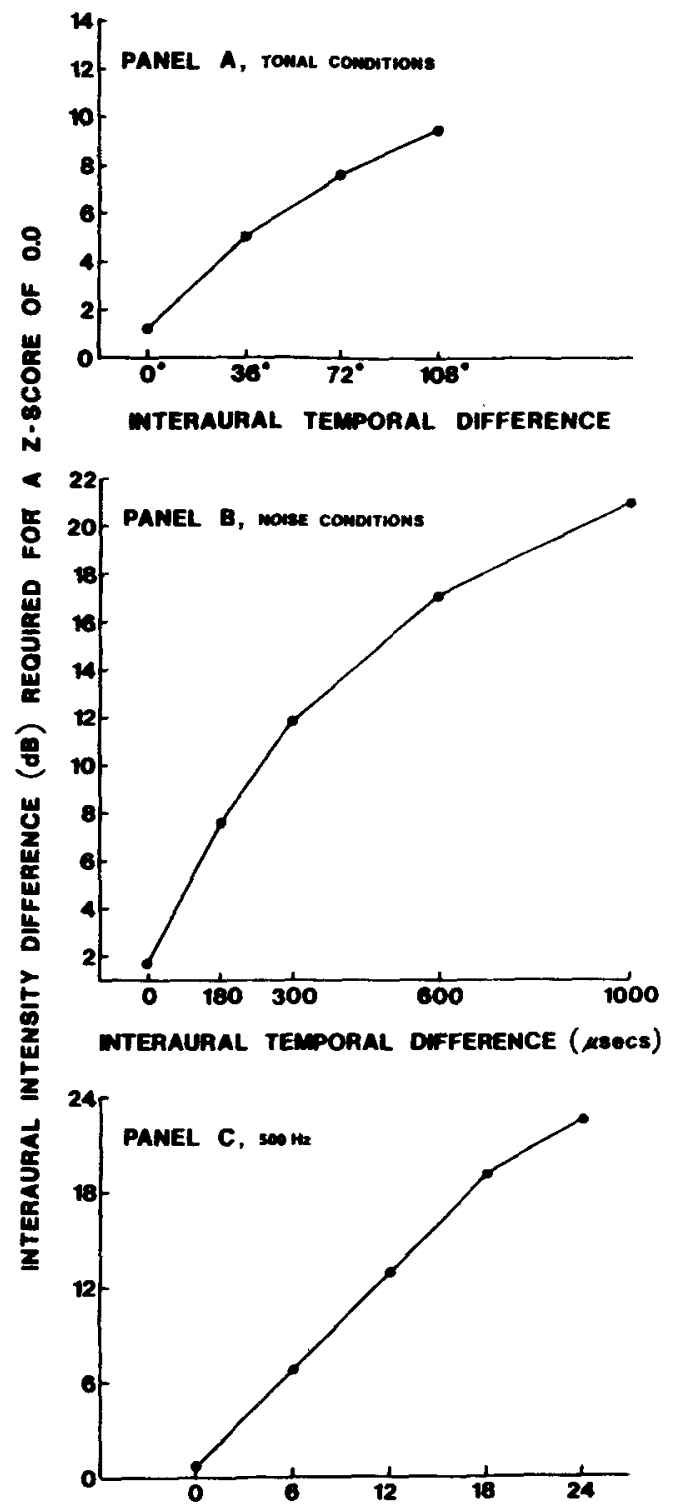

INTERAURAL INTENSITY DIFFERENCE (dB)

Figure 7. The interaural intensive difference required for a $z$ ("right") of 0 as a function of the interaural temporal difference for the average of the tonal data (Panel A), as a function of the interaural temporal difference for the average of the noise data (Panel B), and as a function of the interaural intensive difference (Panel C).

z-score functions in Figures 2, 3, 4, and 5 decrease as the ITD increases. For both the tonal and noise stimuli, the data in Table 1 show that a progressively smaller change in the IID was required for the lateral images to occupy similar locations as the values of the ITD increased from $0^{\circ}$ or $0 \mu \mathrm{sec}$. This suggests that the lateral images associated with larger values of the ITD are less distinct and blend together more than those associated with small values of the ITD. 
Table 1

The Values of the Slope of the Best-Fitting Linear Equation to the Functions Which Relate the Proportion of "Right" Judgments (Expressed in z Scores) to the Interaural Intensive Difference

\begin{tabular}{|c|c|c|c|c|c|}
\hline \multicolumn{6}{|c|}{ Interaural Temporal Difference (Deg) } \\
\hline Condition & 0 & 36 & 72 & 108 & \\
\hline \multirow[t]{3}{*}{ Tones* } & .56 & .46 & .34 & .23 & \\
\hline & \multicolumn{5}{|c|}{ Interaural Temporal Difference (microsec) } \\
\hline & 0 & 150 & 300 & 600 & 1000 \\
\hline \multirow[t]{3}{*}{ Noise** } & .89 & .46 & .54 & .38 & .25 \\
\hline & \multicolumn{5}{|c|}{ Interaural Intensive Difference (dB) } \\
\hline & 0 & 6 & 12 & 18 & 24 \\
\hline $500-\mathrm{Hz}$ Tone & .65 & .70 & .60 & .48 & .45 \\
\hline
\end{tabular}

*Average of the 500. and 1000-Hz and 55. and 65-dB SPL conditions.

**Average of the 55-and 65-dB SPL conditions.

The experiments described in this paper have attempted to demonstrate the interaction between interaural time and interaural intensity in terms of relative lateral position judgments. Various investigators (Sayers. 1964; Teas, 1962; von Békésy, 1969b; Watson \& Mittler. 1965; Yost, 1973) have asked observers to directly indicate where in lateral space an image occurs as a function of changes in IID or ITD. The experiment conducted by Yost (1973) illustrates the general type of method and results. In this experiment. one observer was presented a $200-\mathrm{Hz}$ tone with either an IID or an ITD. The stimulus to be judged was a train of 13100 -msec tones $(50 \%$ duty cycle). The first 5 stimuli in the train were presented at $60 \mathrm{~dB}$ SPL with no interaural differences and, as such, served as a referent condition. The next 8 stimuli were presented at $65 \mathrm{~dB}$ SPL with either an IID or an ITD. The observer marked on a data sheet (premarked with a diagram of the head) which of 21 positions the last 8 (more intense) stimuli occupied in his head. The results from the study are shown in Figure 8 . The data points represent the average of eight presentations, and the vertical bars the range. The data from the first two presentations at each value of IID and ITD were not averaged since they were used for practice.

Both the results and the procedure are consistent with the other investigations. ${ }^{2}$ Considerable variability is obtained in lateral position, especially as the image moves away from midline. The variability is such that any accurate determination of the relationship between IID or ITD and lateral location is difficult, if not impossible. This variability is largest near the outside the head. As Hershkowitz and Durlach (1969b) indicated, headphone placement is a crucial variable under these conditions, since small changes in headphone placement will introduce additional values of IID and ITD which could move the image to the other side of the head. The variability is so large that an ITD of $120^{\circ}$ and an IID of between 6 and $12 \mathrm{~dB}$ could have equivalent lateral locations. This direct method of establishing the interaction of interaural time and intensity does not appear precise.
Figure 8. The lateral position of a perceived image of a $200-\mathrm{Hz}, 65 \cdot \mathrm{dB}$ SPL tone as a function of either an interaural temporal difference (Panel $A$ ) or an interaural intensive difference (Panel B). The data are for one observer. The data points are the mean of eight observations, the vertical bars are the range.

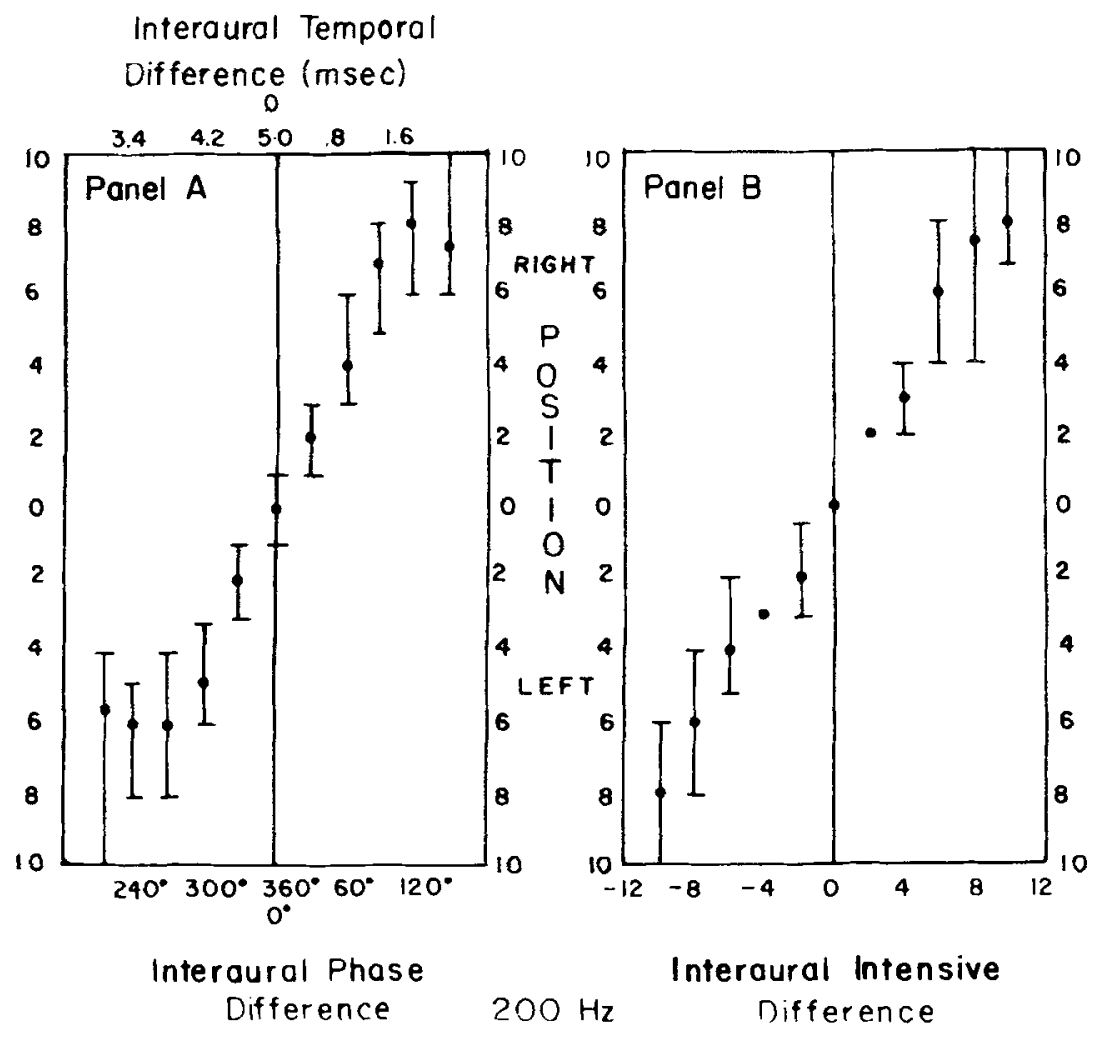


The data shown in Figure 8, however, imply that for sinusoids the lateral image is displaced a decreasing amount of spatial distance as the interaural temporal difference increases from zero degrees. These data also imply that the image appears to be displaced approximately an equal distance for each decibel increase in the interaural intensive difference. If these are valid descriptions of the displacement of lateral images as a function of the IID and the ITD, then the data of Figure 7, Panels $A$ and $C$, are consistent with these descriptions of the displacement of images in lateral space. That is, if the IID does displace the lateral image an equal distance for equal decibel change, then the IID can be used to determine the relative displacement of an image. The data in Figure 7, Panel $\mathrm{C}$, show a constancy between an IID increase and the value of the IID required to obtain an equal spatial location (at least over the same 12-dB range as shown in Figure 8). The data in Figure 7, Panel A, show that as the ITD increases, a decreasing amount of IID is required to obtain a lateral image at the same location as that produced by the ITD. If the IID expressed in decibels is an indicator of relative lateral displacement, then these data also imply that the lateral image is displaced a decreasing amount of spatial distance as the ITD increases from zero degrees. If one assumes that the IID for a noise displaces the lateral image an equal distance for an equal decibel change, then the data in Figure 7, Panel B, suggest that the image associated with a low-pass noise is also displaced a decreasing amount of spatial distance as the ITD increases from a zero value of interaural time. These data only suggest the form of the functions relating lateral displacement to the ITD and the IID. The data in Figure 8 are too variable to provide an exact relationship, and one must make many assumptions before using the data from Figure 7 to substantiate the relationship between lateral displacement and the ITD and the IID.

Both Hershkowitz and Durlach (1969a) and Yost (1974) have used a discrimination procedure to study conditions similar to those investigated in this study. ${ }^{3}$ Yost (1974) studied the lateral discrimination between two values of interaural time. The IID and ITD were varied in the experiments by Hershkowitz and Durlach (1969a). In both studies, the observers were asked to discriminate between two stimuli: the standard stimulus was presented with either an IID or an ITD; the comparison stimulus was presented with the same value of the IID or the ITD as the first stimulus plus an additional value of either the IID or the ITD. The value of the additional interaural difference required for $75 \%$ correct discrimination was the dependent variable in these experiments. The results of these two studies as well as from the present study demonstrate that discriminations involving the $I T D$ are more difficult when the lateral images are away from the midline than when they are near midline.

\section{REFERENCES}

Deatherage, B. H. Examination of binaural interaction. Journal of the Acoustical Society of America, 1966, 39, 232-249. Green, D. M., \& Henning, G. B. Audition. In Annual review of psychology (Vol. 20). Palo Alto, Calif: Annual Reviews, 1969. Pp. 105-128.

Hafter, E. R., \& CArrier, S. C. Binaural interaction in low-frequency stimuli: The inability to trade time and intensity completely. Journal of the Acoustical Society of America, 1972. 51, 1852-1862.

Hafter, E. R., \& JefrRess, L. A. Two image lateralization of tones and clicks. Journal of the Acoustical Society of America, 1968, 44, 563-569.

Hershyowitz, R. M., \& Durlach, N. I. Interaural time and amplitude jnds for a $500-\mathrm{Hz}$ tone. Joumal of the Acoustical Society of America, 1969, 46, 1464-1467. (a)

Hershkowitz, R. M., \& Durlach, N. I. An unsuccessful attempt to determine tradability of interaural time and intensity. Journal of the Acoustical Society of America, $1969,46,583$ (L). (b)

Molino, J. A. Psychophysical verification on predicted interaural differences in localizing distant sound sources. Journal of the Acoustical Society of America, 1974, 55, 139-147.

SAyers, M. B. Acoustic-image lateralization judgments with binaural tones. Journal of the Acoustical Society of America, $1964,36,923.926$.

SAYERS, M. B., \& CherRY, E. C. Mechanism of binaural fusion in the hearing of speech. Joumal of the Acoustical Society of America, 1957, 29, 973-986.

TEAS, D. C. Lateralization of acoustic transients. Journal of the Acoustical Society of America, 1962, 34, 1460-1465.

VON BÉKÉsY, G. Experiments in hearing. New York: McGraw-Hill, 1960.

Watson, C. S., \& Mrtrer. B. T. Time-intensity equivalence in auditory lateralization: A graphical method. Psychonomic Science, 1965, 2, 219-220.

Yost, W. A. Binaural hearing. Communication Sciences Quarterly Report, 1973, 11, 36.

YoST, W. A. Interaural phase-discrimination. Journal of the Acoustical Society of America, 1974, 55, 1294-1304.

\section{NOTES}

1. Although a delay line was used to present the ITD, the 10-msec rise-decay time probably reduces the effect of any onset time cues. Thus, for the tonal conditions, it is probably appropriate to translate the delay-line temporal values into degrees of interaural phase to describe ITD. Throughout this paper, therefore, ITD will refer to either interaural time or to the interaural phase difference corresponding to the value of the delay-line temporal value.

2. The data from Yost's (1973) study suggest that the image moves from midline toward one ear as the ITD increases from 0 to 180 deg. Sayers (1964) argues that the image moves from midline toward one ear as the ITD increases from 0 to $90 \mathrm{deg}$ and then back to midline as ITD increases further from 90 to $180 \mathrm{deg}$. The data in Sayers (1964) Figure 2 show why this appears to happen for his observers. Past an ITD of approximately $120 \mathrm{deg}$, his observers sometimes indicated that images were on the opposite side of the head. For an ITD of $180 \mathrm{deg}$, many observers said that the image was at midline. Yost found that both of these "judgment errors" could be eliminated if (1) great care was used in positioning the observer's headphones, and (2) the observer was provided a referent condition for each judgment. Sayers did not use these precautions. and his data are far more variable than those obtained by Yost. 
3. The lateral left-right judgment procedure is similar to a discrimmation task. In the discrimination task, observers would be asked to indicate (with feedback) which of two stimuli (first or second) was presented with the ITD. Everything else in the procedure could be the same as in the left-right lateralization task. The discrimination procedure was not used because of the potential confounding caused by changes in the intensity at one ear (monarual intensity) produced by presentation of the IID. That is. there is a change (between the two stimuli) in the intensity at one ear which is correlated with which stimulus contains the IID. Thus, observers might use this monaural intensive difference (between the two stimuli) rather than the interaural intensity as their cue for detection.

(Received for publication December 1974; revision received March 18, 1975.) 